Lack of Rating Scale Normalization and a Socioeconomically Advantaged Population Limits the Generalizability of Preadolescent

\section{Transgender Findings}

The article by Olson et $\mathrm{al}^{1}$ exploring the mental health of preadolescent transgender children residing in families that are supportive of their identity attempted to address questions Olson recently raised in a Clinical Perspectives piece published in the Journal of the American Academy of Child and Adolescent Psychiatry. ${ }^{2}$ We read the current article in Pediatrics with great interest, and their effort was laudable. An enhanced understanding of the symptoms and phenomenology of transgender preadolescents is critical, as this material could inform interventions for this marginalized population. Olson et al ambitiously examined symptoms of anxiety and depression in both preschool-aged and early school-aged children. Their approach, however, which included a wide developmental swathe combined with a narrow socioeconomic sample, raises concerns about the meaning and generalizability of their findings.

One challenge when looking at depression and anxiety in preadolescents, particularly in preschool-aged children, is having adequate tools with sufficient validity and reliability to study these symptoms. In the investigation by Olson et al, ${ }^{1}$ transgender children between the ages of 3 and 12 years were compared with age-matched control subjects. The child participants were not surveyed; their parents completed proxy assessments by using the Patient Reported Outcomes Measurement Information System developed by the National Institutes of Health. We noted that the institutes, in their description of the psychometric properties of this tool, indicated that this proxy scale for depression and anxiety is validated only for use in children between the ages of 5 and 17 years. ${ }^{3}$ Olson et al, however, used this instrument across their entire cohort, with $30 \%$ of their study population between the ages of 3 and 5 years. This method means that data from approximately one-third of the sample were from an age group in whom the scale had not been validated, which is problematic in terms of interpretation of the results.

In addition, we noted that the population studied, although ethnically diverse, was heavily skewed to a more financially advantaged group. We observed that $44 \%$ of families had a household income greater than $\$ 125$ 000. This income level was above the 80th percentile for the nation, which starts at $\$ 100000 .{ }^{4}$ Given the linkage that exists between lower socioeconomic standing and poorer outcomes in children's mental health, ${ }^{5}$ we wondered what the results would be if a sample that was more representative of the nation were used. Might the lack of detected psychopathology be related as much to the wealth and privilege of the families observed?

Overall, we commend Olson et $\mathrm{al}^{1}$ on their effort to bring data to bear about our understanding of this understudied group. As they do, we remain curious regarding the relationship, if any, between transgender and psychopathology of childhood, particularly in preschoolaged children. We would advocate, however, for utilization of normalized scales in looking at the age under study and for identifying a population that more accurately reflects the clinical population most children's mental health providers typically serve.

Alastair J. McKean, MD Child and Adolescent Psychiatrist, Assistant Professor of Psychiatry, Mayo Clinic E-mail: mckean.alastair@mayo.edu

Jennifer L. Vande Voort, MD

Child and Adolescent Psychiatrist, Assistant Professor of Psychiatry, Mayo Clinic
Paul E. Croarkin, D0 Child and Adolescent Psychiatrist, Assistant Professor of Psychiatry, Mayo Clinic

POTENTIAL CONFLICT OF INTEREST: The authors have indicated they have no potential conflicts of interest to disclose.

\section{REFERENCES}

1. Olson KR, Durwood L, DeMeules M, McLaughlin KA. Mental health of transgender children who are supported in their identities. Pediatrics. 2016;137(3): e20153223

2. Olson KR. Prepubescent transgender children: what we do and do not know. J Am Acad Child Adolesc Psychiatry. 2016;55(3): 155-156.e3

3. Varni JW, Thissen D, Stucky BD, et al. PROMIS $^{\circledast}$ Parent Proxy Report Scales for children ages 5-7 years: an item response theory analysis of differential item functioning across age groups. Qual Life Res. 2014;23(1):349-361

4. DeNavas-Walt C, Proctor BD. Income and Poverty in the United States: 2014-Current Population Reports. Washington, DC: US Department of Commerce; 2015

5. Ashford J, Smit F, van Lier PA, Cuijpers P, Koot HM. Early risk indicators of internalizing problems in late childhood: a 9-year longitudinal study. J Child Psychol Psychiatry. 2008;49(7):774-780

doi:10.1542/peds.2016-1203A

\section{Author Response to McKean, Vande Voort, and Croarkin (2016)}

We are grateful for the comment from McKean et al on our article reporting on anxiety and depression among a group of socially transitioned, prepubescent transgender children ages 3 to 12 years. ${ }^{1}$ Although we have additional research in progress that can better speak to some of their concerns (eg, the focus in the first article on parental reports), we are able to provide additional information from the Pediatrics data set to address their 2 major concerns: (1) the (high) socioeconomic status of our participants; and (2) the results with 
regard to the age distribution of our sample.

First, McKean et al point out that our study focused on a "narrow socioeconomic sample," or more specifically, that our sample was considerably wealthier than the average American or Canadian family. This issue is one we have discussed elsewhere, ${ }^{2}$ and we agree that it is a limit to the generalizability of our findings. To better understand this issue, we report our results according to socioeconomic group in Table 1. It is noteworthy that the sample of families with incomes less than $\$ 75000$ was small in the Pediatrics article. ${ }^{1}$ We have since recruited additional lower income families that will appear in a future article, and we appreciate that McKean et al reiterated the importance of this issue. We do generally find, across all groups of participants, that children in families earning more than $\$ 125000$ per year exhibit lower mean levels of anxiety and depression, and this finding may be especially true for transgender children.

A second concern raised by McKean et al involved our use of a "wide developmental swathe"; that is, children between the ages of 3 and 12 years. They also noted that our measure was used with 3-year-olds $(n=1)$ and 4-year-olds $(n=8)$ in addition to children aged $\geq 5$ years ( $n=64)$, for whom the scale was designed. Although breaking the sample into subgroups resulted in small sample sizes, we nonetheless did so. This approach allowed us to also compare our responses with a group of gender nonconforming children reported by Singh et al. ${ }^{3}$ Importantly, the article by Singh et al reported on gender-dysphoric children at the Toronto clinic who came from high-income backgrounds. Although the exact measure used across the 2 articles was different (Patient Reported Outcomes Measurement Information System versus the Child Behavior Checklist), the scores on both measures are based on large normed samples that use the standardized $t$ scores. In general, we found a lower mean internalizing score in our Pediatrics sample (52.2, the average $t$ score of the depression and anxiety Patient Reported Outcomes Measurement Information System scales) than Singh et al did (58.4, on the internalizing subscale of the Child Behavior Checklist). In addition, at every age, we found fewer children in the "clinical range" (top 10\% of internalizing scores): 3- to 5-yearolds, $4.5 \%$ of our 22 children and $23 \%$ of 139 children from Singh et al; 6- to 7-year-olds, $6.3 \%$ of our 16 children and $47 \%$ of 68 children from Singh et al; 8- to 9-year-olds, $8.7 \%$ of our 23 children and $64.4 \%$ of 45 children from Singh et al; and 10- to 12 -year-olds, $16.7 \%$ of our 12 children and $77.7 \%$ of 18 children from Singh et al. Our mean internalizing scores according to age

TABLE 1 Mean $t$ Score Values for Anxiety and Depression for Participants in Olson et al ${ }^{1}$ According to Household Income

\begin{tabular}{lccc}
\hline Income Level & Controls & Siblings & Transgender \\
\hline$<\$ 75000$ & & & 55.9 \\
$\quad$ Anxiety & 51.1 & 56.2 & 51.2 \\
Depression & 49.9 & 49.2 & 11 \\
$\quad$ Sample Size & 14 & 5 & 56.8 \\
$>\$ 75,00>$ and $<\$ 125000$ & & & 53.3 \\
Anxiety & 52 & 53.2 & 30 \\
Depression & 49.3 & 49.8 & \\
Sample Size & 31 & 19 & 51.2 \\
$>\$ 125000$ & & & 46.8 \\
Anxiety & 49.5 & 50.8 & 32 \\
Depression & 46.6 & 48.9 & 25 \\
$\quad$ Sample Size & 28 & & 30 \\
\hline
\end{tabular}

were as follows: 3 - to 5-year-olds, 50.2; 6- to 7-year-olds, 52.9; 8- to 9-year-olds, 51.0; and 10- to 12-yearolds, 56.9. Means according to age group are unavailable in Singh et al.

Reporting these results next to each other allows us to address the question by McKean et al of our study: "Might the lack of detected psychopathology be related as much to the wealth and privilege of the families observed?" Given that Singh et $\mathrm{al}^{3}$ also worked with gender nonconforming children who came from high-income backgrounds, and those children had high rates of internalizing disorders, we do not believe that high wealth and privilege alone can explain our findings. Although the samples differed in other ways (eg, Canadians versus primarily Americans, the years when the studies were run), we believe that 2 key features distinguished our sample from theirs: (1) all of our participants claim a gender identity that differs from their sex; and (2) our participants socially transitioned to present as that gender in everyday life.

We greatly appreciate the interest in our study, and we hope that our future research as well as research from other laboratories and clinics can ultimately address the critical questions raised by McKean et al: do we see good mental health outcomes in transgender children who socially transition early in development who are from lower income backgrounds? Do we see similar rates of psychopathology when we use reporters other than parents? We believe that as the number of children socially transitioning early in development increases, and as more research groups study this population, we will have more definitive answers to these questions.

Kristina R. Olson
Associate Professor of Psychology, University of
Washington
E-mail: krolson@uw.edu
Lily Durwood
Research Assistant
Research Assistant 
Madeleine DeMeules Research Study Coordinator

Katie A. McLaughlin Assistant Professor of Psychology

POTENTIAL CONFLICT OF INTEREST: The authors have indicated they have no potential conflicts of interest to disclose.

\section{REFERENCES}

1. Olson KR, Durwood L, DeMeules M, McLaughlin KA. Mental health of transgender children who are supported in their identities. Pediatrics. 2016;137(3): e20153223

2. Olson KR, McLaughlin KA. How to raise happy, healthy transgender kids. LA Times. 2016; February 26
3. Singh D, Bradley SJ, Zucker KJ. Commentary on "An affirmative intervention for families with gender variant children: parental ratings of child mental health and gender" by Hill, Menvielle, Sica, and Johnson (2010). J Sex Marital Ther. 2011;37(2):151-157, discussion 158-160

doi:10.1542/peds.2016-1203B 